\title{
Attentional capture by signals of reward persists following outcome devaluation
}

\author{
Poppy Watson, Yenti Pavri, Jenny Le, Daniel Pearson, and Mike E. Le Pelley \\ School of Psychology, University of New South Wales, Sydney, New South Wales 2052, Australia
}

\begin{abstract}
Attention, the mechanism that prioritizes stimuli in the environment for further processing, plays an important role in behavioral choice. In the present study, we investigated the automatic orienting of attention to cues that signal reward. Such attentional capture occurs despite negative consequences, and we investigated whether this counterproductive and reflexive behavior would persist following outcome devaluation. Thirsty participants completed a visual search task in which the color of a distractor stimulus in the search display signaled whether participants would earn water or potato chips for making a rapid eye movement to a diamond target, but looking at the colored distractor was punished by omission of the signaled reward. Nevertheless, participants looked at the water-signaling distractor more frequently than the chipsignaling distractor. Half the participants then drank water ad libitum before continuing with the visual search task. Although the water was now significantly less desirable for half of the participants, there was no difference between groups in the tendency for the water-signaling distractor to capture attention. These findings suggest that once established, counterproductive attentional bias to signals of reward persists even when those outcomes are no longer valuable. This suggests a "habit-like" attentional mechanism that prioritizes reward stimuli in the environment for further action, regardless of whether those stimuli are aligned with current goals or currently desired.
\end{abstract}

[Supplemental material is available for this article.]

Habitual responses are argued to underlie many maladaptive behaviors, including overconsumption of food (Everitt and Robbins 2005; Verhoeven and de Wit 2018). Attention, the mechanism that prioritizes stimuli in the environment for further processing, plays an important role in behavioral choice and is the first step in selecting stimuli for action (Krajbich and Rangel 2011; Cavanagh et al. 2014; Smith and Krajbich 2018; Pearson et al. 2022). However, little is known about the role that attentional processes may play in supporting habitual behavior (Jiang and Sisk 2019).

Years of research have demonstrated that several different factors influence what we attend to (for reviews, see Awh et al. 2012; Le Pelley et al. 2016; Watson et al. 2019a). Stimuli that stand out on the basis of their physical characteristics (luminance, color, etc.) tend to capture attention in an involuntary manner (Theeuwes 1992; Theeuwes et al. 2003). Attention can also be directed toward stimuli with certain features on the basis of current task goals (e.g., when searching for a favorite brand of ice cream at the supermarket, particular colors and shapes that are on the packaging will be prioritized) (Folk et al. 1992; Yantis 2000). Furthermore, stimuli that have previously been associated with motivationally salient outcomes (e.g., rewards or punishments) are also more likely to receive attentional priority relative to stimuli with similar physical characteristics but that have not been associated with motivationally salient outcomes (Anderson et al. 2011; Le Pelley et al. 2015; Pearson et al. 2015; Schmidt et al. 2015; Anderson and Britton 2019; Mikhael et al. 2021). A number of studies have established that attentional orienting to signals of reward can be involuntary and occurs even when doing so leads to negative outcomes (e.g., loss of monetary bonuses) (Le Pelley et al. 2015, 2017; for review, see Watson et al. 2019a). This involuntary attentional capture by reward is fast and reflexive, with eye-tracking studies demonstrat- ing that rapid eye movements are particularly likely to be directed (erroneously) toward reward-related distractors (Pearson et al. 2016; Watson et al. 2020).

As for other types of rewards, several studies have demonstrated attentional bias toward signals of food and drink rewards that are currently desired (Rose et al. 2013, 2018; Watson et al. 2021). For example, Watson et al. (2021) used a food version of the valuemodulated attentional capture (VMAC) task. In this eye-tracking protocol, which was also used in the present study, participants were instructed to make an eye movement (saccade) to a diamondshaped target on each trial to earn different food and drink rewards. A colored circle in the display indicated which outcome (e.g., salty potato chips or water) was available in that trial. For example, a blue circle might signal that chips were available, and an orange circle might signal that water was available (with these color-reward relationships counterbalanced across participants; all other shapes in the display were gray). Participants were instructed that if any gaze was registered on the colored circle, then the reward would be omitted in that trial. Participants' desire for the different rewards was manipulated by having hungry and thirsty participants either eat potato chips or drink water ad libitum before completing the food VMAC task. Replicating the pattern of results previously observed with monetary rewards (Failing et al. 2015; Le Pelley et al. 2015; Pearson et al. 2015), participants looked more often at the distractor that signaled the currently valuable (nonsated) food/drink outcome than the distractor signaling the less valuable (sated) outcome. Critically the reward-related attentional bias that was observed was counterproductive because there was a negative consequence-the more often participants looked at the valuable-food/drink distractor, the more they missed out 
on earning that reward for consumption. Participants were never trained to look at the food-signaling distractors; instead, looking at the distractor was always punished with reward omission. Analyses taking into account first saccade latencies demonstrated that the reward-related attentional bias (tendency to look at the valuable-food- relative to the devalued-food-signaling distractor) was particularly pronounced among the fastest saccades, suggesting that - as has been observed in studies for monetary rewardthe bias to attend to valuable food outcomes is automatic and reflexive (Pearson et al. 2016; Le Pelley et al. 2019; Watson et al. 2021).

Findings of counterproductive attentional capture by stimuli signaling food rewards, when hungry, align with data showing that cues predictive of motivationally relevant outcomes (such as monetary reward) can involuntarily capture attention (for review, see Watson et al. 2019a). However, what about when signaled outcomes are not motivationally relevant? Just as other behaviors can become habitual and be triggered reflexively by environmental stimuli (Dickinson 1985; de Wit and Dickinson 2009; Robbins and Costa 2017), the automatic attentional prioritization of stimuli that signaled previously valuable food and drink outcomes might persist, even when those outcomes are no longer valuable. That is, once attentional bias for a food-associated stimulus has been established, does it persist when that food is no longer desirable?

Habitual responses are developed through behavioral repetition and triggered directly by environmental cues and contexts, even when the associated outcomes are no longer desired (Dickinson 1985; de Wit and Dickinson 2009; Robbins and Costa 2017). In the field of associative learning, outcome devaluation following a period of training is the gold-standard procedure for assessing whether behavior is controlled by the value of the outcome that it produces or instead is controlled by stimuli that signal the availability of that outcome irrespective of its value (Dickinson 1985). In the context of instrumental learning, for example, a rat might first be trained on a task in which a certain response will earn a particular outcome (e.g., pressing the lever will earn a food pellet). The outcome is then devalued through either specific satiation (being fed food pellets to satiety) or through taste aversion (pairing food pellets with a noxious chemical to induce nausea). Instrumental responses directed toward earning food pellets are then tested in extinction (to prevent any further learning). Behavior is said to be goal-directed if the animal can integrate the new knowledge about the changed outcome value with the instrumental response-outcome knowledge, as evidenced by reduced responding for the devalued outcome, as compared with a control group of rats for whom the outcome has not undergone devaluation and hence remains valuable. In contrast, if animals persist with carrying out the response that leads to the devalued outcome, then behavior is said to be habitual and reflexively triggered by the stimulus or context (de Wit and Dickinson 2009). Attentional bias for reward cues is Pavlovian in nature (Bucker and Theeuwes 2017) and does not meet the criteria for an instrumental behavior (because it has been demonstrated to occur despite negative consequences) (Le Pelley et al. 2016). Nonetheless, as has been observed in some animal studies, it is possible that Pavlovian orienting responses toward a food-associated stimulus can become reflexively triggered by the stimulus itself, even if the signaled outcome is not currently desired (Morrison et al. 2015; Patitucci et al. 2016). In other words, once established, the attentional prioritization may become "habit-like" and be persistently triggered by rewardsignaling stimuli even if the signaled outcomes are no longer valuable (Jiang and Sisk 2019).

Research investigating the effect of postconditioning changes in outcome value on attentional bias for food has been reported previously (Pool et al. 2014; De Tommaso et al. 2017; De Tommaso and Turatto 2021). These studies used an initial condi- tioning phase in which participants were trained to associate one image (termed the CS+ image) with the delivery of a pleasant chocolate odor (Pool et al. 2014) or high probability of drink reward (De Tommaso et al. 2017; De Tommaso and Turatto 2021), and another image with no odor/low probability of drink reward (termed the CS- image). Attentional bias to the images was then assessed during a subsequent (unrewarded) test phase that used a spatial cueing task: In each trial, both images appeared simultaneously, and a target-to which participants had to respond-then appeared in the location of one of the images. It was found that, for participants who were hungry (Pool et al. 2014) or thirsty (De Tommaso et al. 2017; De Tommaso and Turatto 2021) throughout the task, responses in the test phase were faster when the target appeared in the location of the CS+ image versus the CS- image, consistent with the idea of an attentional bias to the reward-signaling cue. The question of more interest is whether the magnitude of this reward-related attentional bias reduces if, following conditioning, the motivational value of the reward is reduced, or whether participants continue to show a bias even if the signaled outcome is no longer desirable. The existing evidence on this issue is mixed. Pool et al. (2014) found some evidence to suggest that the attentional bias to the chocolate-signaling CS+ (vs. the CS-) was reduced in participants who were sated on chocolate following conditioning (but prior to the attentional test) relative to participants who were not sated. These findings are consistent with the idea that the attentional bias to the CS+ is mediated by a representation of the current value of the signaled reward. In contrast, informal between-experiments comparisons based on the data reported by De Tommaso and colleagues (De Tommaso et al. 2017; De Tommaso and Turatto 2021) suggested that the size of the bias toward the signal of the drink reward was similar whether or not participants had been sated on the drink immediately following the initial conditioning phase, and hence no longer desired this drink during the test phase. These latter findings are thus consistent with the idea that attentional prioritization of a color previously associated with a drink reward (i.e., faster responding in trials where the target appeared in the drink-related color) persists even when the drink outcome is no longer desired.

Notwithstanding the inconsistent findings, there were some limitations to these studies that place constraints on the conclusions that can be drawn about the persistence of reward-related attention bias following outcome devaluation. Specifically, in the test phase of these aforementioned experiments, there was no cost to participants for prioritizing attention to the CS+ image (Pool et al. 2014; De Tommaso et al. 2017; De Tommaso and Turatto 2021). In all of these studies, the location in which the target appeared during the test phase was determined independently of the location in which the cues appeared. This meant that the target was equally likely to appear in the location of the CS+ as it was to appear in any other specific location. As a result, this procedure produces no conflict between task goals (quickly locate the target) and attentional prioritization of reward-related cues. Consequently, any attentional bias that is observed may reflect strategic prioritization of cues that previously provided useful information and/or were previously associated with reward: While there was no benefit to pursuing this strategy, there was also no cost to doing so. Participants in the study of Pool et al. (2014) reported liking the CS+ image more than the CS- image following the conditioning phase. Following a conditioning protocol of this type, therefore, participants may continue to attend to the (preferred) $\mathrm{CS}+$ in a top-down fashion during the subsequent test phase even in the absence of reward. Under these conditions, it is thus unclear whether the observed effect of reward reflects the operation of an automatic and reflexive attentional process versus a more strategic, top-down mechanism. Given that the test of attentional bias does not distinguish between automatic and 
strategic processes, we cannot know the level at which any effect (or lack of effect) of devaluation is mediated. This may in turn have contributed to the mixed findings of previous studies with regard to effects of devaluation: Since the attentional test that was used does not provide a selective measure of automatic and reflexive effects of reward on attention, differences in top-down strategy across studies (perhaps due to differences in the reward values used or clarity of instructions to participants, etc.) may have resulted in different patterns of behavior on test.

Our aim in the present study was to provide a more diagnostic measure of the influence of outcome devaluation on (specifically) reflexive and automatic attentional capture by food-and-drinkrelated stimuli; that is, on the way in which these reward-signaling stimuli can take control over our behavior. For this purpose, we used the food VMAC task described earlier. In this procedure, there is an explicit cost to attending to reward-signaling distractors, since this results in omission of reward, and so the task puts attentional prioritization of reward-related cues in opposition to task goals. Under these conditions, an attentional bias toward rewardsignaling cues provides an index of the effect of reward on automatic attentional processes, and hence use of the food VMAC task allows us to assess whether reflexive and counterproductive attentional capture by food-and-drink-related stimuli persists or reduces following outcome devaluation. An additional advantage of the food VMAC task is its use of eye tracking, which provides a relatively direct and "online" measure of moment-by-moment shifts of attention over the course of a trial (given that eye movements and attention are tightly coupled) (e.g., see Deubel and Schneider 1996). In contrast to the indirect, response-time-based measures used in previous studies in this area (Pool et al. 2014; De Tommaso et al. 2017; De Tommaso and Turatto 2021), eye tracking allows us to examine more closely when capture by reward-related stimuli is likely to occur. For example, by analyzing direction of eye movements as a function of saccade latency, we can investigate whether faster saccades are more likely to go in the direction of reward-related distractors (cf. Pearson et al. 2016; Le Pelley et al. 2019; Watson et al. 2021)—a pattern that might be expected of a reflexive behavior.

In the present study, participants completed the food VMAC task in which colored distractors signaled the availability of potato chips or water in each trial. To earn the signaled outcome, participants had to move their eyes quickly to a diamond target without first looking at the colored distractor. We prefed all participants salty potato chips before they performed the initial, training phase of the food VMAC task, which was intended to establish baseline attentional prioritization for a valuable relative to a not-valuable outcome: Based on previous work (Watson et al. 2021), we expected to find evidence of greater attentional capture by the watersignaling distractor than the chip-signaling distractor during this training phase. After the training phase, half of the participants drank water ad libitum, before all participants completed another session of the food VMAC task conducted in nominal extinction (participants were aware that they were still earning outcomes but were not informed in each trial as to what outcome was earned). Nominal extinction is similar to the traditional extinction test used to investigate habits following outcome devaluation in animals (Dickinson 1985) and ensures that behavior is based on previously learned associations, independent of any impact of experience of the delivery of the (de)valued outcome itself. However, whereas nominal extinction ensures that participants do not experience outcomes during the test phase and thus prevents further learning, it also means that (human) participants are still motivated to respond during the task (Hogarth and Chase 2011; Watson et al. 2014). During this critical test phase, water was still valuable for half the participants (relative to chips), but neither water nor chips were valuable for the other half of participants. Based on prior research, we hypothesized two potential patterns of results. On the one hand, some reflexive responses to food-signaling cues (such as pupil dilation in humans or approach behavior in animals) are reported to be sensitive to outcome devaluation (Lelos et al. 2011; Pool et al. 2019), which would lead to predictions that following water devaluation, attention would no longer be captured automatically by the water-signaling distractor, and hence that the magnitude of attentional bias toward water would be reduced in participants who had undergone water devaluation relative to those who had not (cf. Pool et al. 2014). On the other hand, just as behavioral responses can become habitual and be triggered reflexively by environmental stimuli (Robbins and Costa 2017), involuntary attentional orienting to the water-signaling distractor might persist even if the water is no longer desirable (cf. De Tommaso et al. 2017; De Tommaso and Turatto 2021). In this case, we would expect that attentional bias to the water-signaling distractor would remain similar in both groups of participants regardless of whether water had been devalued or not.

\section{Results}

\section{Demographics, questionnaires, and consumption}

Table 1 shows demographic information for the 64 participants included in the final sample (32 in the water-devaluation group and 32 in the water-valuable group). Due to technical error, BMI measurements from two participants were missing. The groups did not differ significantly at baseline on any of the measures.

\section{Desire ratings: time 1 vs. time 2}

After time 1 (baseline), all participants ate potato chips to satiation during the first TV watching (chip-devaluation) phase. Desire ratings at time 1 (baseline) and time 2 (after eating chips) are shown separately for the two groups in Figure 1A; these data were analyzed using ANOVA with within-subject factors of outcome (chips/water), time point (time 1 before/time 2 after eating chips), and

Table 1. Baseline measures for the water-valuable and water-devalued groups.

\begin{tabular}{lccc}
\hline & Water devalued $(\boldsymbol{n = 3 2 )}$ & Water valuable $(\boldsymbol{n = 3 2})$ & Comparison \\
\hline Age & $20.1(0.4)$ & $19.8(0.4)$ & $t(62)=0.48, P=0.630$ \\
Sex ratio (male:female) & $11: 21$ & $14: 18$ & $X^{2}(1)=0.59, P=0.442$ \\
BMI & $23.1(0.6)$ & $21.5(0.6)$ & $t(60)=1.9, P=0.063$ \\
Baseline hunger & $59(4)$ & $62(4)$ & $t(62)=0.47, P=0.637$ \\
Baseline thirst & $71(4)$ & $67(3)$ & $t(62)=0.49, P=0.626$ \\
Baseline desire chips & $64(4)$ & $76(4)$ & $t(62)=0.53, P=0.599$ \\
Baseline desire water & $75(4)$ & $2.6(0.1)$ & $t(62)=0.28, P=0.780$ \\
DEBQ restrained eating & $2.6(0.2)$ & $t(62)=0.24, P=0.814$ \\
\hline
\end{tabular}

Note: Rows other than that for sex ratio show mean (SEM) for the water-devalued and water-valuable groups. The final column shows the results of independent $t$-tests comparing the two groups ( $\chi^{2}$ test in the case of the sex ratio). (BMI) Body mass index, (DEBQ) Dutch Eating Behavior Questionnaire. 
A

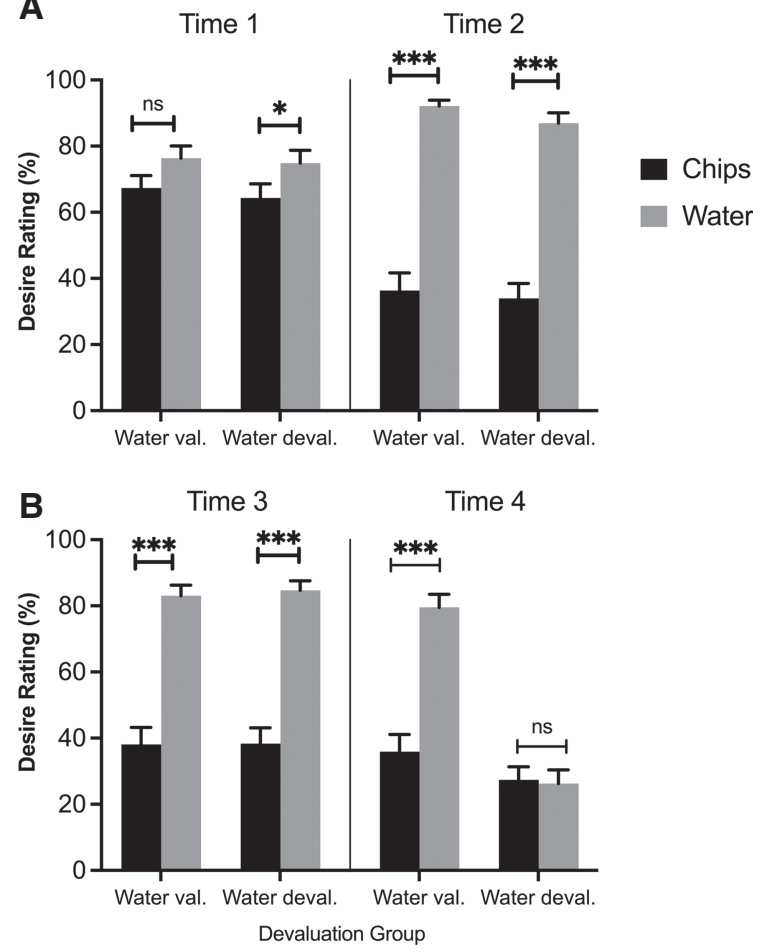

Figure 1. Desire ratings for chips and water at various time points throughout the experiment, shown separately for the group that was sated on water during the water-devaluation phase (water-devalued group) and the group that was not given the opportunity to drink water at all during the experiment (water-valuable group). $(A)$ Desire ratings before (time 1) and after (time 2) the first TV watching phase, during which all participants ate potato chips. (B) Desire ratings before (time 3 ) and after (time 4) the second TV watching phase, during which time only the water-devalued group was given the opportunity to drink water ad libitum. Error bars represent SEM.

between-subject factor of devaluation group (water-devalued vs. water-valuable group). As expected, there was an interaction between time point and outcome $\left(F_{(1,62)}=107.2, P<0.001, \eta_{p}{ }^{2}=\right.$ $0.63)$. At time 1 (baseline), participants had a slight preference for water, which increased considerably after eating chips. Unsurprisingly, given that the two groups had thus far had identical experiences during the experiment, there was no main effect of devaluation group $\left(F<1, P=0.341, \eta_{p}{ }^{2}=0.02\right)$ or interactions involving devaluation group ( $\left.F s<1, P s>0.626, \eta_{p}{ }^{2}<0.004\right)$. These analyses confirm that overall, when entering the training phase of the food VMAC task, participants desired the water significantly more than the chips.

\section{Desire ratings: time 3 vs. time 4}

Desire ratings at time 3 (after completing the training phase of the food VMAC task) and time 4 (after watching TV for the second time, before the test phase of the task) are shown in Figure 1B and were analyzed via ANOVA with factors of outcome (chips/water), time point (time 3 before/time 4 after water manipulation), and devaluation group (water-devalued vs. water-valuable group). As expected, given that only the water-devalued group was able to consume water to satiation between $\mathrm{T} 3$ and T4, there was a three-way interaction $\left(F_{(1,62)}=37.8, P<0.001, \eta_{p}{ }^{2}=0.38\right)$. To explore this interaction, we ran a separate two-way ANOVA for each devaluation group. Overall, participants in the water-valuable group had elevated desire for water over chips at both T3 and T4, as indicated by a main effect of outcome $\left(F_{(1,31)}=63.9, P<0.001, \eta_{p}{ }^{2}=\right.$ $0.67)$. There were no further significant effects $(F \mathrm{~s}<2.5, P \mathrm{~s}>0.122$, $\left.\eta_{p}{ }^{2}<0.08\right)$. In contrast, the water-devaluation group showed the expected interaction between time point and outcome $\left(F_{(1,31)}=\right.$ 51.4, $\left.P<0.001, \eta_{p}{ }^{2}=0.624\right)$. At T3, participants in the waterdevaluation group desired water significantly more than chips $\left(t_{(31)}=7.8, P<0.001, d=1.4\right)$. However, after consuming water during the second TV watching phase, there was no significant difference in desire ratings for chips versus water $\left(t_{(31)}=0.3, P=0.755\right.$, $d=0.06$ ). These analyses confirm that overall, for participants in both groups, the elevated desire for water persisted through the training phase of the food VMAC task. However, when entering the test phase of the food VMAC task, the water-devaluation group had been successfully sated on water, and only the water-valuable group still desired water significantly more than chips.

\section{Food VMAC task: training phase}

Primary analysis of data from the training and test phases of the food VMAC task, reported here, was based on frequentist ANOVA. Corresponding analyses using Bayesian ANOVA are in the Supplemental Material, with inclusion Bayes factors (using the "effects across matched models" approach) (van den Bergh et al. 2020; https://www.cogsci.nl/blog/interpreting-bayesianrepeated-measures-in-jasp) also reported here. In all cases, findings of the Bayesian analysis mirror those of the frequentist analysis.

The proportion of omission trials (i.e., trials in which participants looked at the colored distractor, hence causing omission of the food/drink outcome) in the training phase of the food VMAC task was examined using ANOVA with within-subject factor of distractor type (chips vs. water) and between-subject variable of devaluation group. In line with the desire ratings, demonstrating that overall participants had elevated preference for water over chips during the training phase of the food VMAC task, participants had their attention captured more frequently by the water distractor relative to the chips distractor during the training phase, therefore missing out on more water than chip points $\left(F_{(1,62)}=14.99, P<\right.$ $\left.0.001, \eta_{p}{ }^{2}=0.20, \mathrm{BF}_{\text {inclusion }}=205\right)($ see Fig. $2 \mathrm{~A})$. There was no significant main effect of devaluation group $\left(F<1, P=0.626, \eta_{p}{ }^{2}=0.04\right.$, $\left.\mathrm{BF}_{\text {inclusion }}=0.334\right)$ or interaction $\left(F<1, P=0.641, \eta_{p}{ }^{2}=0.004\right.$, $\mathrm{BF}_{\text {inclusion }}=0.268$ ).

We also investigated the likelihood of the first saccade on each trial going to the distractor, as a function of saccade latency. Briefly, for each participant, we calculated the direction and latency of the first saccade on each trial and then ranked these from fastest to slowest (separately for the training phase vs. test phase and chips distractor vs. water distractor trials). We then split these saccade latencies into three time bins (defined by tertiles of the distribution). Attentional capture as a function of first saccade latency was then examined by analyzing the proportion of saccades that went in the direction of the distractor. For the training phase data, we used ANOVA with variables of distractor type (chip vs. water distractor), saccade latency time bin (fastest, middle, and slowest thirds) and devaluation group. There was a significant main effect of distractor type $\left(F_{(1,40)}=10.2, \quad P=0.003, \quad \eta_{p}{ }^{2}=0.20\right.$, $\mathrm{BF}_{\text {inclusion }}=5738$ ): As can be seen in Figure 2, B (water-valuable group) and $\mathrm{C}$ (water-devalued group), first saccades were more likely to go to the water distractor than the chip distractor. There was also a main effect of saccade latency time bin $\left(F_{(2,80)}=99.67\right.$, $\left.P<0.001, \eta_{p}{ }^{2}=0.71, \mathrm{BF}_{\text {inclusion }}=5.025 \times 10^{23}\right)$, with the proportion of saccades to the distractor increasing with faster saccades. There was also an interaction between these two variables $\left(F_{(2,80)}=4.32\right.$, $\left.P=0.020, \eta_{p}{ }^{2}=0.10, \mathrm{BF}_{\text {inclusion }}=0.699\right)$, with increased attentional capture by the water relative to the chip distractor at the shortest saccade latencies. Notably, when examining data from the fastest latency time bin, first saccades were significantly more likely to go 
toward the water distractor than the chip distractor $\left(t_{(41)}=2.4, P=0.021, d_{z}=0.37\right)$. The ANOVA did not reveal any significant effects involving devaluation group $\left(F \mathrm{~s}<1, P \mathrm{~s}>0.513, \eta_{p}{ }^{2}<0.02, \mathrm{BF}_{\text {inclusion }}<\right.$ 0.289 ).

\section{Food VMAC task: test phase}

Although the groups differed in their current desire for water when entering the test phase, the pattern of gaze during this phase was similar in both groups (see Fig. 2D-F). ANOVA analysis of the proportion of omission trials revealed a main effect of distractor type, with participants looking significantly more often at the water distractor than the chip distractor during the test phase $\left(F_{(1,62)}=\right.$ $10.45, P=0.002, \eta_{p}{ }^{2}=0.14, \mathrm{BF}_{\text {inclusion }}=$ 27.9). Critically, however, there was no main effect of devaluation group $(F<1$, $P=0.575, \eta_{p}{ }^{2}=0.005, \mathrm{BF}_{\text {inclusion }}=0.230$ ) or interaction between devaluation group and distractor type $\left(F<1, P=0.536, \eta_{p}{ }^{2}=\right.$ $0.006, \mathrm{BF}_{\text {inclusion }}=0.253$ ) (see Fig. 2D). The nonsignificant interaction shows that the devaluation manipulation did not have a significant effect on participants' pattern of attentional bias. A follow-up analysis examined the more specific (one-tailed) prediction made by an account in which the attentional bias was determined by current outcome value; namely, that the magnitude of the bias during the test phase would be smaller in the group that had undergone devaluation of water relative to the group that had not. For each participant, we calculated a food VMAC score, given by water omissions minus chip omissions. An independent sample $t$-test revealed that food VMAC scores were not significantly lower in the water-devalued group than the water-valuable group $\left(t_{(62)}=0.62, P=\right.$ 0.268 [one-tailed], $d=0.15$ ), with the corresponding one-tailed Bayes factor confirming moderate evidence for the null hypothesis $\left(B F_{O-}=5.84\right.$, error $\left.<0.005 \%\right)$. These results indicate that reduced desire for water did not result in a reduction in automatic orienting to a cue signaling water reward.

ANOVA analysis of attentional capture by reward-signaling distractors as a function of first saccade latency during the test phase revealed a main effect of distractor type $\left(F_{(1,40)}=9.42, P=\right.$ $\left.0.004, \eta_{p}{ }^{2}=0.19, \mathrm{BF}_{\text {inclusion }}=13,000\right)$, with first saccades being generally more likely to go to the water distractor than the chip distractor (see Fig. 2E [water-valuable group], F [water-devalued group]). There was also a main effect of saccade latency time bin $\left(F_{(2,80)}=\right.$ $\left.115.74, P<0.001, \eta_{p}{ }^{2}=0.74, \mathrm{BF}_{\text {inclusion }}=1.21 \times 10^{21}\right)$. The interaction between these two variables did not reach significance $\left(F_{(2,80)}=2.65, \quad P=0.083, \quad \eta_{p}^{2}=0.06, \quad B F_{\text {inclusion }}=0.309\right)$; notably (and as for the training phase), the saccades in the fastest time bin were significantly more likely to go to the water than the
D Test Phase

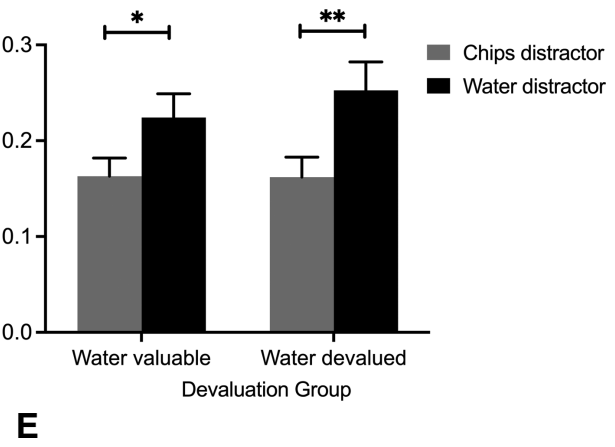

Test Phase

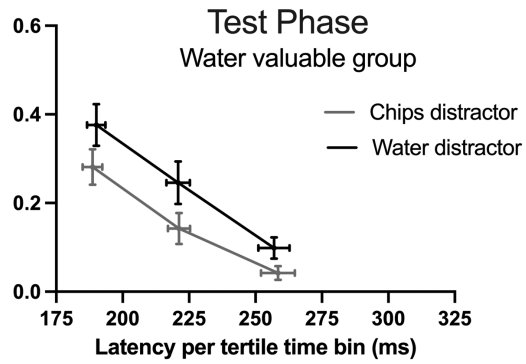

$\mathbf{F}$

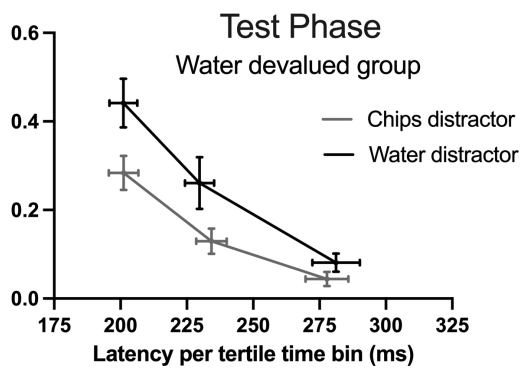

Figure 2. Eye-tracking data from the food VMAC task. The left panels depict performance during the training phase of the task, during which time both groups reported more desire for water than chips. The right panels depict performance during the test phase of the task, during which time participants in the water-devalued group no longer valued water over chips. (A) Training phase: proportion of omissions (trials where participants looked at the distractor and missed out on the signaled reward) when the search display contained a chip distractor versus a water distractor for the water-valuable and waterdevalued groups. (B) Training phase: proportion of first saccades in the direction of the chips and water distractor as a function of saccade latency for the water valuable group. Mean first saccade participant saccade latency distributions. Symbols on each line represent the fastest, middle, and slowest saccade latency time bins. (C) Training phase: proportion of first saccades in the direction of the chips and water distractor as a function of saccade latency for the water-devalued group. $D-F$ depict the corresponding data from the test phase. Error bars represent SEM.

chip distractor $\left(t_{(41)}=2.74, P=0.009, d_{z}=0.42\right)$. Finally, there were no significant effects involving devaluation group $(F \mathrm{~s}<1, P \mathrm{~s}$ $\left.>0.475, \eta_{p}{ }^{2}<0.02, \mathrm{BF}_{\text {inclusion }}<0.232\right)$. Follow-up analyses with data from the fastest time bin (where attentional biases were most pronounced) were used to assess the one-tailed hypothesis of whether the bias toward the water distractor was reduced in the water-devalued group relative to the water-valuable group. We calculated bias scores for this fastest time bin (proportion of first saccades in the direction of the water distractor minus the chip distractor). An independent sample $t$-test revealed that bias scores were not significantly lower in the water-devalued group than the water-valuable group $\left(t_{(40)}=0.49, P=0.314\right.$ [one-tailed], 
$d=0.15$ ), with the corresponding one-tailed Bayes factor providing moderate evidence for the null hypothesis $\left(B F_{O-}=4.98\right.$, error $<$ $0.005 \%)$. These findings indicate that devaluation of water did not result in a reduction in rapid orienting to the water-signaling distractor (relative to the chip-signaling distractor), even though desire for water had been sated in the water-devalued group.

\section{Discussion}

In the present study, we investigated whether the tendency to automatically orient attention to distractors signaling food and drink outcomes would persist even if those outcomes were no longer desirable. Participants completed the food VMAC task twice. During the first (training) phase of the task, participants desired water more than chips and showed a tendency to orient attention more frequently to the cue signaling that water was available, with this pattern being most pronounced among the fastest eye movements that participants made. This occurred even though this behavior was counterproductive, since it led to the cancelation of the water reward (replicating the findings of Watson et al. 2021). Participants in the water-devalued group were then offered water ad libitum, thereby sating their desire for water such that these participants no longer desired water more than chips. However, in a subsequent test phase of the food VMAC task, this manipulation did not result in a shift in the attentional bias for water (relative to chips) compared with participants in the water-valuable group, who did not undergo devaluation of water. Both the watervaluable and water-devalued groups showed persistent orienting of attention to the water cue relative to the cue signaling chips, particularly for the most rapid saccades.

One possible interpretation of the results from this experiment could be that the satiation manipulation affected only the current desire for the water outcome ("wanting") and that the attentional bias, being unaffected, was indexing something more global, such as overall "liking" for the water (Robinson and Berridge 1993; Berridge et al. 2009). However, data from previous experiments using preconditioning satiation manipulations have demonstrated consistency in self-reported desire for foods on the one hand and attentional bias on the other (Rose et al. 2013, 2018; De Tommaso et al. 2017; Watson et al. 2021). In these studies, with a pretraining satiation manipulation, attentional prioritization developed only to cues signaling currently valuable rewards. In a study by Watson et al. (2021), for example, regardless of whether participants were sated on chips, water, chocolate, or salty popcorn prior to the eye-tracking task, attentional capture by the distractor signaling the devalued outcome was always significantly less than capture by the valued distractor. These findings clearly demonstrate that the satiation procedure has the potential to change the attentional priority of a reward-associated stimulus. In the present experiment, however, satiation had no effect on attention when it came after the distractor-outcome associations had already been established: We did not observe reduced attentional orienting to the cue that signaled the now-devalued water outcome during the test phase (for participants in the water-devalued group). The only thing that differed between these studies was the timing of the satiation manipulation. The implication is that the development of attentional prioritization during training depends on the current value of the experienced outcomes (as shown by Watson et al. 2021), but that this training results in a persistent bias that is insensitive to a subsequent shift in outcome value (as shown in the present study).

Our finding of insensitivity to postconditioning outcome devaluation is consistent with prior findings reported by De Tommaso and colleagues (De Tommaso et al. 2017; De Tommaso and Turatto 2021), but conflicts with the devaluation sensitivity reported by Pool et al. (2014). Notably, the present study used a procedure in which the attentional bias was explicitly counterproductive, and hence provided a more diagnostic demonstration that (specifically) reflexive and automatic attentional capture by food-and-drink-related stimuli is insensitive to outcome devaluation. Others have highlighted the association between attention and subsequent food choice (Danner et al. 2016; Smith and Krajbich 2018; Zoltak et al. 2018; Peschel et al. 2019), suggesting that an initial attentional prioritization of (subsequently devalued) food stimuli could promote overt, behavioral responses directed at those foods (for discussion of the relationship between automatic attentional prioritization and subsequent decision making, see Pearson et al. 2022).

As mentioned above, the orienting of attention to cues that signal reward in the VMAC task is not instrumental. Instead, it is largely Pavlovian in nature, and behavior in the VMAC task has been likened to sign-tracking behavior in animals (Albertella et al. 2019a; Colaizzi et al. 2020). Sign tracking refers to a tendency of some animals to approach and engage with cues that signal reward in a Pavlovian conditioning protocol. For example, when the insertion of a lever signals that food will be delivered to a food magazine, some animals (goal trackers) will learn to approach the location of food delivery. In contrast, sign trackers will develop Pavlovian approach behaviors to the lever (and may begin sniffing or nibbling it) even if this means that they miss out on the food reward (Hearst and Jenkins 1974). Some studies have reported that sign tracking to the reward-signaling cue persists even when the associated outcome has been devalued (similar to what was observed in the present study) (Morrison et al. 2015; Patitucci et al. 2016). However, others have reported that sign-tracking behavior is reduced when the outcomes signaled by the cues are no longer desired (Derman et al. 2018; Maria-Rios et al. 2021). Differences in the strength of outcome-devaluation manipulations and the length of Pavlovian training have been proposed for these differences in the sensitivity of sign tracking to outcome devaluation observed across studies (Amaya et al. 2020; Keefer et al. 2020). Further work is needed to elucidate the factors influencing (in)sensitivity of sign tracking to outcome devaluation in both humans and animals, particularly given the potential importance of sign-tracking behavior as a behavioral marker for addiction vulnerability (Tomie 2018; Albertella et al. 2019a; Colaizzi et al. 2020).

There were some limitations to the present study that should be noted. First, we did not include a condition where participants drank water before the training phase and then ate chips to satiation during the devaluation phase. A demonstration of the persistence of attentional bias to potato chips following chip devaluation would have strengthened the ecological validity of the study. We have previously demonstrated that attentional bias for valuable relative to devalued food rewards can be established, regardless of whether potato chips, water, chocolate, or salty popcorn functioned as the devalued or valued distractor (Watson et al. 2021). As such, as proof of concept and for simplicity's sake, we focused the present study on the condition where water was devalued following training. We assume that the same results would be observed with other food and drink outcomes and that attentional orienting to a calorie-dense junk food would be particularly resistant to outcome devaluation once established. However, this is something that future studies will have to assess. Second, we note that power during the saccade latency analysis dropped significantly following participant exclusions. Accordingly, while the pattern of results from the saccade latency analysis is fully in line with the non-latency-based analyses, these findings should be interpreted with caution.

It is worth also considering alternative explanations for the observed pattern of results. The test phase of the experiment was conducted in nominal extinction, which ensured that participants did not have exposure to (devalued) outcomes during the test 
phase while providing motivation to still try to perform the task correctly (Hogarth and Chase 2011). However, it is possible that participants paid attention to the cues during the test phase because they continued to have informational value (i.e., they still signaled the available reward on each trial) (Gottlieb 2012). Against this notion, previous experiments suggest that informational value does not drive the attentional prioritization of cues signaling highreward stimuli because this behavior has been seen to persist even when participants are aware that rewards are no longer available and thus the cues no longer have any informational value (Anderson et al. 2011; Mine and Saiki 2015; Anderson and Halpern 2017; Albertella et al. 2019b; Watson et al. 2019b; for review, see Anderson 2016). Another possibility associated with the use of the nominal extinction procedure is that participants may have been unsure which outcome (chips or water) was associated with a particular color during the test phase, or they may have simply lost motivation to perform the task. The pattern of results speaks against these possibilities, however, because we would expect some fluctuation in the pattern of eye gaze data if participants were suddenly unable to discriminate between the available rewards in each trial (or if lack of reward induced noncompliance). Figure 2, B-F, shows that there is little change in the distribution of saccade latencies from the training phase to the test phase (and no increase in saccade latencies that would be expected if participants lost motivation during the test phase).

Finally, it is possible that various context shifts that occurred during the experiment may have influenced the pattern of results. Studies in animals have suggested that behavior that is insensitive to outcome devaluation is highly context-dependent and that subtle changes in context after outcome devaluation can bring goal-directed control back "online" (Bouton 2021). This raises the possibility that if the test phase of the present experiment had been conducted in a context completely different from the training phase then we may have observed elimination of the persistent "habit-like" orienting to the devalued distractor. Of particular relevance to the current experiment, Amaya et al. (2020) also argued that the studies that failed to observe devaluation effects on sign-tracking behavior did so because they conducted devaluation in a context different from the behavioral task. Extrapolating to the current experiment, it may be that if the water satiation had occurred in the same room as the eye-tracking task, the shift in value would have led to a corresponding shift in the attentional bias. However, it remains unclear whether these context effects play a role in human outcome-devaluation paradigms (Watson and de Wit 2018). This feeds into a wider discussion of what exactly constitutes a context switch in human experiments (Rosas et al. 2013) and is an interesting avenue for future research to explore.

In summary, the results of the present study highlight that after outcome devaluation, attention continues to be captured by cues that signaled previously desirable rewards, despite the fact that the signaled outcome is no longer motivationally relevant. The food VMAC paradigm proved to be a useful tool for examining the integration of motivational and cognitive factors underlying behavior. There is a clear link between attentional prioritization of foods and increased likelihood of choosing those foods (Danner et al. 2016; Smith and Krajbich 2018; Zoltak et al. 2018; Peschel et al. 2019; Gluth et al. 2020), and future studies can examine whether this pervasive attentional bias can support poor food choices, particularly relevant in the context of obesity and overeating.

\section{Materials and Methods}

\section{Participants and apparatus}

This study was approved by the University of New South Wales (UNSW) Sydney Human Research Ethics Advisory Panel
(Psychology). G*Power analysis (Faul et al. 2007) indicated that 60 participants would provide power of $85 \%$ for detecting a significant interaction between devaluation group (water valuable/water devalued) and outcome (chip/water distractor trials) during the test phase, assuming a small-medium effect size $(f=0.2)$. Default settings were used for correlation between repeated-measures $(0.5)$ and nonsphericity correction (1). We aimed to test 70 participants, assuming (on the basis of our previous food VMAC study) (Watson et al. 2021) that there would be a number of participant exclusions. In total, 71 UNSW Sydney students participated in exchange for course credit. Participants were randomly assigned to either the water-devaluation group or water-valuable group. Participants were asked to refrain from eating or drinking $2 \mathrm{~h}$ prior to the study, but five participants admitted to noncompliance with this instruction and were excluded from all analyses. In line with our previous experiments (Watson et al. 2021), one participant was also excluded because they ate $<10 \mathrm{~g}$ of chips during the initial chip-eating phase. One final participant was excluded for not attempting to answer the questionnaires or complete the task properly. The final group therefore consisted of 64 participants (32 in the waterdevaluation group and 32 in the water-valuable group).

All participants were tested individually. Gaze was recorded using a Tobii TX300 eye tracker (with sample rate of $300 \mathrm{~Hz}$ ) mounted on a 23 -in monitor $(1920 \times 1080$ resolution, $60-\mathrm{Hz}$ refresh rate). A chinrest $60 \mathrm{~cm}$ from the screen was used to stabilize head position. For gaze-contingent aspects of stimulus presentation, gaze data were first downsampled to $100 \mathrm{~Hz}$. Stimulus presentation was controlled by MATLAB using Psychophysics Toolbox extensions (Brainard 1997; Pelli 1997; Kleiner et al. 2007). Experiment scripts, raw data, and processed data showing means for individual participants are available via the Open Science Framework at https://osf.io/q9zju.

\section{Design}

\section{Food VMAC task}

During this visual search task (as used by Watson et al. 2021), participants were first instructed to fixate on a cross in the center of the screen (fixation display) (see Fig. 3A). The fixation display turned yellow once $700 \mathrm{msec}$ of gaze time had accumulated in the central fixation region. After a further $300 \mathrm{msec}$, the screen blanked for $150 \mathrm{msec}$ before the search display appeared. The search display consisted of six shapes (each $2.3^{\circ} \times 2.3^{\circ}$ visual angle) distributed evenly around the center-point of the screen, with the center of each shape at an eccentricity of $5.1^{\circ}$. One of these shapes was a diamond (the target), and the remaining five shapes were circles. On each trial, one of the circles (the distractor) was colored either orange (RGB values: 193, 95, 30) or blue (RGB: 37, 141, 165) with similar luminance $\left(24.5 \mathrm{~cd} / \mathrm{m}^{2}\right)$. All other shapes were gray (RGB: 70, 70, 70; luminance $\sim 8.3 \mathrm{~cd} / \mathrm{m}^{2}$ ). Target and distractor locations were determined randomly in each trial. Two gaze measurement areas of interest (AOIs) were defined: one AOI centered on the target with a diameter of $3.5^{\circ}$ visual angle, and a larger AOI centered on the colored distractor circle with a diameter of $5.1^{\circ}$.

In each trial, participants were required to move their eyes as quickly and directly as possible to the diamond target. The color of the distractor circle signaled whether a chip or water point was available in each trial (these points were later converted into real food/drink outcomes). For half of the participants, blue signaled that a chip point was available (the chip distractor), and orange signaled a water point (the water distractor); for the other half of the participants, this mapping was reversed. A rapid eye movement directly to the diamond target earned the participant either a chip or water point, as appropriate. Crucially, however, if any gaze was registered within the distractor AOI, the reward was canceled: Trials in which this occurred were termed "omission trials." The trial was not terminated if gaze was registered on the distractor; instead, all trials ended immediately after a response to the target was registered (100 msec of gaze dwell time on the diamond target AOI), or after $2000 \mathrm{msec}$ (time-out). If response time to the target was $<1000 \mathrm{msec}$ and the trial was not an omission trial (i.e., no gaze was registered on the reward-signaling distractor), 
A

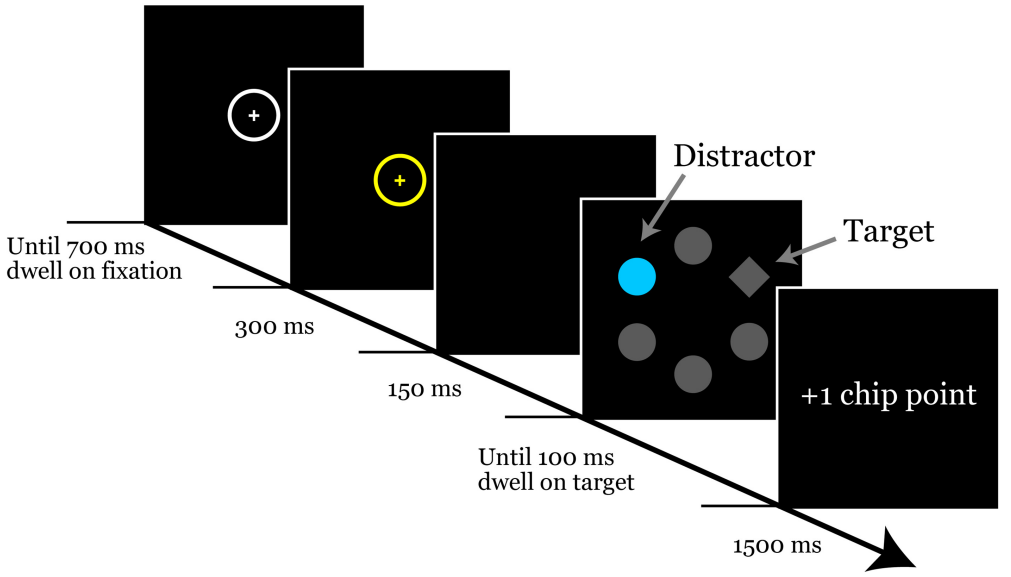

B

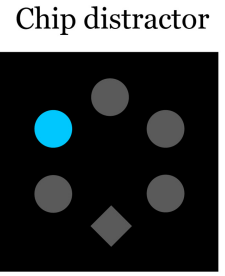

Water distractor

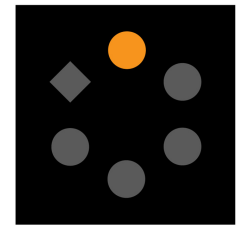

C

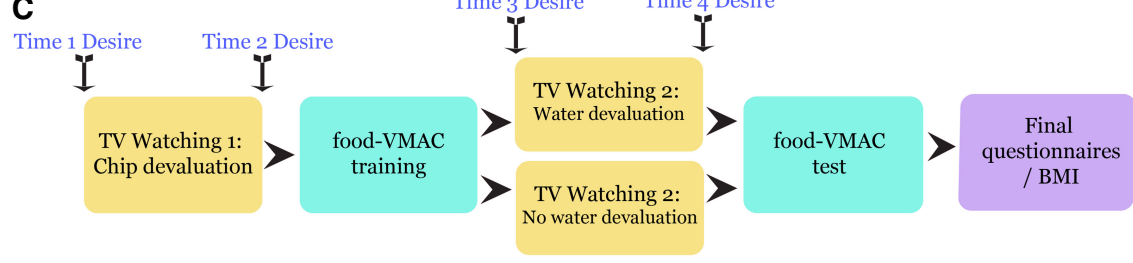

Figure 3. (A) Trial structure of the training phase of the food VMAC task (not to scale). In each trial, participants had to move their eyes to the diamond target to earn reward. They also had to inhibit gaze toward a colored distractor circle, because if they looked at the distractor the reward was canceled. During the test phase, participants were not informed as to the specific type of point earned; instead, the feedback was simply "+1 point" or "+0 points." (B) Schematic examples of search displays for the two different trial types. The color of the distractor signaled whether a chip or water point was available for making a saccade to the diamond target. Here we show an example in which blue signals chips and orange signals water; in reality, relationships between colors and outcomes were counterbalanced across participants. (C) Schematic of the procedure of the experiment. Phases shown in yellow are TV watching phases. During TV watching 1, all participants ate chips ad libitum; during TV watching 2, only participants in the water devaluation group were offered water. The training and test phases of the food VMAC task are depicted in aqua. Points at which desire ratings for chips and water were assessed are indicated (time 1 desire to time 4 desire).

participants saw a feedback screen alerting them to the points that had been won in that trial. During the training phase, this feedback screen indicated whether a chip or water point had been earned (e.g., "+ 1 water point"). During the test phase, this feedback screen simply read " +1 point" (regardless of whether a chip or water distractor was present in the search display). In both phases of the task, if gaze had been registered on the distractor, the trial was an omission trial, and the feedback screen indicated " +0 points." If response time was $>1000 \mathrm{msec}$, feedback read "+0 points, too slow." If no response was registered before the time-out, feedback read: "Too slow, please try to look at the diamond more quickly." During the training phase, in all cases in which participants had not earned a reward, feedback also stated "You could have won 1 chip/water point" (as appropriate). Feedback was presented for $1500 \mathrm{msec}$, and the intertrial interval was $700 \mathrm{msec}$.

\section{Dutch Eating Behavior and Compliance Questionnaire}

To verify that the two groups had similar levels of eating restraint (a factor known to influence attention bias for food rewards using this task) (Watson et al. 2021), the restrained eating scale of the Dutch Eating Behavior Questionnaire (DEBQ) was used (van Strien et al. 1986). This consists of 10 items, such as "Do you deliberately eat less in order to not become heavier?," answered on a five-point scale ranging from never to very often (scored from 1 to 5). Participants were also asked to indicate whether they had complied with instructions to not eat or drink anything in the $2 \mathrm{~h}$ before the study.

\section{Food and drink rewards}

The two rewards used in the experiment were "Smith's Crinkle Cut Plain" potato chips (2290 kJ per $100 \mathrm{~g}$, $556 \mathrm{mg}$ of sodium per $100 \mathrm{~g}$ ) and chilled, filtered tap water. The chips were presented in a large wooden serving bowl containing an entire large packet (170 g; equivalent to approximately nine single-serve [19-g] lunchbox-size snack packs). The water was presented in a large 1.5 -L plastic jug filled to the top, with a disposable cup alongside.

\section{Desire questionnaire}

At four time points during the experiment (time 1: baseline, before chip devaluation; time 2: after chip devaluation; time 3: before water devaluation; and time 4: after water devaluation), participants were asked to consume a small chip/sip of water and then rate their current desire for each item on a visual analog scale (using the computer mouse). The scale ranged from 0 to 100 and was marked with the anchors "no desire" (at the 0 point), "neutral" (at the midpoint), and "strong desire" (at the maximal point). Participants were also asked to rate their current hunger and thirst on similar scales marked with the anchors "not at all," "neutral," and "extremely hungry/thirsty."

\section{$T V$ watching room}

At various time points throughout the experiment, participants were asked to sit in the "TV watching room" and consume either chips or water to satiation. The TV watching room was a darkened laboratory cubicle with a comfortable chair and a computer monitor, intended to induce mindless eating while participants watched a comedy show. In the chip-devaluation phase, the 170-g bowl of chips (but no water) was on the table in front of the participant. During the water-devaluation phase, for participants assigned to the water-devaluation condition, the 1.5-L jug of water (but no chips) was on the table in front of the participant.

\section{Procedure}

A schematic of the procedure is shown in Figure 3C.

\section{$T V$ watching 1: chip-devaluation phase}

Before the participant arrived, the experimenter prepared the jug of water and bowl of chips and weighed each using digital kitchen scales. Participants first completed the informed consent procedure before being taken to the TV watching room. The experimenter left the room while the participant completed the desire 
questionnaire (time 1). The experimenter then explained that the participant would watch TV for $10 \mathrm{~min}$ and that they were expected to try to consume as many chips as possible in that time. The experimenter then left the room, ensuring that the bowl of chips was left for the participant to consume ad libitum (no water was available). After $10 \mathrm{~min}$, the experimenter returned to the TV watching room and asked the participant to complete the desire questionnaire again (time 2). While the participant was completing the training phase of the food VMAC task, the experimenter weighed the remaining chips to calculate the amount consumed.

\section{Food VMAC task: training phase}

Participants then completed the food VMAC task in the eyetracking laboratory (no food was visible). They were informed that their task was to move their eyes to the diamond "as quickly and directly as possible," and that they would receive some chips and water at the end of the experiment, depending on how many chip points and water points they earned (no further information on the conversion rate between points and rewards was provided). Participants were then instructed on the color-reward contingencies in the food VMAC task; for example, that whenever a blue circle was present in the display, they could win one chip point for looking at the diamond, and whenever an orange circle was present, they could win one water point. Participants were also informed that looking at the colored distractor would result in the reward for that trial being omitted. Participants were asked to repeat these instructions to the experimenter to ensure that they had been understood.

Participants then completed six blocks, each containing 15 trials with a chip distractor and 15 trials with a water distractor, in random order (180 trials total). Participants took a self-paced break after every two blocks. The training phase of the food VMAC task took 25 min to complete.

\section{TV watching 2: water-devaluation phase}

Participants were taken back to the TV watching room. The experimenter left the room while the participant completed the desire questionnaire (time 3). The experimenter then explained that the participant would watch the rest of the TV show for $10 \mathrm{~min}$. Those in the water-valuable group were not given any further instructions (and no food or drink was visible for this group). Participants in the water-devaluation group were told that they were expected to try to consume as much water as possible in the $10 \mathrm{~min}$ from a $1.5-\mathrm{L}$ jug placed in front of them. No chips were available. After $10 \mathrm{~min}$, the experimenter returned to the TV watching room and asked the participant to complete the desire questionnaire again (time 4). While the participant was completing the test phase of the food VMAC task, the experimenter weighed the remaining water to calculate the amount consumed (for those in the water-devaluation group).

\section{Food VMAC task: test phase}

Participants then returned to the food VMAC task in the eyetracking laboratory (no food or drink was visible). Participants were informed that the task was the same as before (to look quickly and directly at the diamond), but that now they would not be told in each trial what outcome point (water or chips) they had won. They were instructed that they were still earning water and chip points and that they would receive these at the end of the experiment (nominal extinction) (Hogarth and Chase 2011; Watson et al. 2014)

Participants then completed six blocks in the test phase, each block containing 15 trials with a chip distractor and 15 trials with a water distractor, in random order (180 trials total). The task was the same as during the training phase, only that instead of receiving feedback for each trial as to what type of point (water point or chip point) had been won, participants saw " +1 point" instead. Participants took a self-paced break after every two blocks. The test phase of the food VMAC task took 25 min to complete.

\section{Final questionnaires and measurements}

Finally, participants completed the Dutch Eating Behavior and Compliance Questionnaire. Height and weight measurements were then recorded for body mass index (BMI) calculations. Participants were then debriefed and received their water and chip winnings from the task in the form of paper cups filled with water and 30-g snack packets of potato chips. Participants received one, two, three, or four cups of water (bags of chips), depending on whether their final number of water (chips) points was above $0 \%$, $25 \%, 50 \%$, or $75 \%$ of the maximum number of points that could have been earned. The entire experiment took $\sim 75 \mathrm{~min}$ to complete.

\section{Data processing}

The processed mean data for each participant, used in the analyses reported below, are available at https://osf.io/q9zju.

Processing of behavioral data from the food VMAC task followed our standard procedures (e.g., Pearson et al. 2016; Le Pelley et al. 2019). Data were discarded from the first two trials of each block, trials that timed out before a response was recorded ( $1.7 \%$ of all trials), and trials with $<25 \%$ valid gaze location data $(0.2 \%$ of all trials). For the remaining trials, valid gaze location data were recorded in $M=99 \%$ (SEM $=0.3 \%$ ) of samples from the eye tracker. Our primary measure of interest was the proportion of trials in which gaze was detected on the colored distractor, leading to omission of the point that had been available, calculated separately for trials featuring the chip distractor or water distractor.

In line with our previous work (Pearson et al. 2016; Le Pelley et al. 2019; Watson et al. 2021), we also investigated the likelihood of the first saccade in each trial going to the distractor, as a function of saccade latency. Previous findings that the fastest saccades are those that are more likely to go in the direction of the high-reward distractor (relative to the low-reward distractor) are indicative of the reflexive nature of the attentional bias. Using the raw data from the eye tracker (sampled at $300 \mathrm{~Hz}$ ), a velocity threshold identification algorithm (Salvucci and Goldberg 2000) was used to identify saccades. Following linear interpolation across gaps in the raw gaze data $<75 \mathrm{msec}$, the data were smoothed with a fivepoint moving average filter. The first saccade in each trial was defined as the first eye movement with a velocity criterion of $40^{\circ}$ visual angle per second that remained above this threshold for at least $10 \mathrm{msec}$. The saccade was classified as being in the direction of a particular stimulus location if the saccade vector had an angular deviation $<30^{\circ}$ to the left or right of the center of that stimulus (see Pearson et al. 2016). Trials were excluded from this analysis if the start point of the identified saccade was $>100$ pixels from the center of the screen, if it was an anticipatory saccade (defined as a saccade latency $<80 \mathrm{msec}$ ), or if there was insufficient gaze data to identify a saccade. In line with our previous work, we excluded 22 participants from the saccade analysis because they had $>20 \%$ of total trials excluded from either phase of the food VMAC task. For the remaining 42 participants ( 22 in the water-devalued condition and 20 in the water-valuable condition), $7.8 \%$ of total trials were discarded. For each participant and each phase of the task (training phase vs. test phase), we used the Vincentizing procedure (Ratcliff 1979) to separate the remaining saccade latency data into three time bins, representing the fastest, middle, and slowest groups of saccades separately for the water- and chip-distractor trials (Le Pelley et al. 2019; Watson et al. 2021). In other words, trial data for each participant were grouped by task phase and signaled outcome, first saccade latencies were then ranked from fastest to slowest, and finally these ranked latencies were split into three time bins defined by tertiles of the distribution. For both phases of the experiment (training and test), in each time bin of the chipdistractor and water-distractor trials, we calculated the proportion of first saccades that went in the direction of the distractor. 


\section{Ethical statement}

The experiment reported here was approved by the UNSW Sydney Human Research Ethics Advisory Panel (Psychology) with file number HREAP 2957. Full written informed consent procedures were used.

\section{Competing interest statement}

The authors declare no competing interests.

\section{Acknowledgments}

This work was supported by an Australian Research Council Discovery Early Career Researcher Award to P.W. (DE200100591), and by an Australian Research Council Discovery Project (DP200101314)

\section{References}

Albertella L, Chamberlain SR, Le Pelley ME, Greenwood L-M, Lee RSC, Den Ouden L, Segrave RA, Grant JE, Yücel M. 2019a. Compulsivity is measurable across distinct psychiatric symptom domains and is associated with familial risk and reward-related attentional capture. CNS Spectr 25: 519-526. doi:10.1017/S1092852919001330

Albertella L, Watson P, Yücel M, Le Pelley ME. 2019b. Persistence of value-modulated attentional capture is associated with risky alcohol use. Addict Behav Rep 10: 100195. doi:10.1016/j.abrep.2019.100195

Amaya KA, Stott JJ, Smith KS. 2020. Sign-tracking behavior is sensitive to outcome devaluation in a devaluation context-dependent manner: implications for analyzing habitual behavior. Learn Mem 27: 136-149. doi:10.1101/lm.051144.119

Anderson BA. 2016. The attention habit: how reward learning shapes attentional selection. Ann N Y Acad Sci 1369: 24-39. doi:10.1111/nyas .12957

Anderson BA, Britton MK. 2019. On the automaticity of attentional orienting to threatening stimuli. Emotion 20: 1109-1112. doi:10.1037/ emo0000596

Anderson BA, Halpern M. 2017. On the value-dependence of value-driven attentional capture. Atten Percept Psychophys 79: 1001-1011. doi:10 .3758/s13414-017-1289-6

Anderson BA, Laurent PA, Yantis S. 2011. Learned value magnifies salience-based attentional capture. PLOS ONE 6: e27926. doi:10.1371/ journal.pone.0027926

Awh E, Belopolsky AV, Theeuwes J. 2012. Top-down versus bottom-up attentional control: a failed theoretical dichotomy. Trends Cogn Sci 16: 437-443. doi:10.1016/j.tics.2012.06.010

Berridge KC, Robinson TE, Aldridge JW. 2009. Dissecting components of reward: 'liking,' 'wanting,' and learning. Curr Opin Pharmacol 9: 65-73. doi:10.1016/j.coph.2008.12.014

Bouton ME. 2021. Context, attention, and the switch between habit and goal-direction in behavior. Learn Behav 49: 349-362. doi:10.3758/ s13420-021-00488-z

Brainard DH. 1997. The psychophysics toolbox. Spat Vis 10: 433-436. doi:10.1163/156856897X00357

Bucker B, Theeuwes J. 2017. Pavlovian reward learning underlies value driven attentional capture. Atten Percept Psychophys 79: 415-428. doi:10 .3758/s13414-016-1241-1

Cavanagh JF, Wiecki TV, Kochar A, Frank MJ. 2014. Eye tracking and pupillometry are indicators of dissociable latent decision processes. J Exp Psychol Gen 143: 1476-1488. doi:10.1037/a0035813

Colaizzi JM, Flagel SB, Joyner MA, Gearhardt AN, Stewart JL, Paulus MP. 2020. Mapping sign-tracking and goal-tracking onto human behaviors. Neurosci Biobehav Rev 111: 84-94. doi:10.1016/j.neubiorev.2020.01.018

Danner L, de Antoni N, Gere A, Sipos L, Kovács S, Dürrschmid K. 2016. Make a choice! Visual attention and choice behaviour in multialternative food choice situations. Acta Alimentaria 45: 515-524. doi:10.1556/066.2016 .1111

Derman RC, Schneider K, Juarez S, Delamater AR. 2018. Sign-tracking is an expectancy-mediated behavior that relies on prediction error mechanisms. Learn Mem 25: 550-563. doi:10.1101/lm.047365.118

De Tommaso M, Turatto M. 2021. On the resilience of reward cues attentional salience to reward devaluation, time, incentive learning, and contingency remapping. Behav Neurosci 135: 389-401. doi:10.1037/ bne 0000423

De Tommaso M, Mastropasqua T, Turatto M. 2017. The salience of a reward cue can outlast reward devaluation. Behav Neurosci 131: 226-234. doi:10 $.1037 /$ bne0000193
Deubel H, Schneider WX. 1996. Saccade target selection and object recognition: evidence for a common attentional mechanism. Vis Res 36: 1827-1837. doi:10.1016/0042-6989(95)00294-4

de Wit S, Dickinson A. 2009. Associative theories of goal-directed behaviour: a case for animal-human translational models. Psychol Res 73: 463-476. doi:10.1007/s00426-009-0230-6

Dickinson A. 1985. Actions and habits: the development of behavioural autonomy. Philos Trans R Soc Lond B Biol Sci 308: 67-78. doi:10.1098/ rstb.1985.0010

Everitt BJ, Robbins TW. 2005. Neural systems of reinforcement for drug addiction: from actions to habits to compulsion. Nat Neurosci 8: 1481 1489. doi:10.1038/nn1579

Failing M, Nissens T, Pearson D, Le Pelley M, Theeuwes J. 2015. Oculomotor capture by stimuli that signal the availability of reward. J Neurophysiol 114: 2316-2327. doi:10.1152/jn.00441.2015

Faul F, Erdfelder E, Lang A-G, Buchner A. 2007. G*Power 3: a flexible statistical power analysis program for the social, behavioral, and biomedical sciences. Behav Res Methods 39: 175-191. doi:10.3758/ bf03193146

Folk CL, Remington RW, Johnston JC. 1992. Involuntary covert orienting is contingent on attentional control settings. J Exp Psychol Hum Percept Perform 18: 1030-1044. doi:10.1037//0096-1523.18.4.1030

Gluth S, Kern N, Kortmann M, Vitali CL. 2020. Value-based attention but not divisive normalization influences decisions with multiple alternatives. Nat Hum Behav 4: 634-645. doi:10.1038/ s41562-020-0822-0

Gottlieb J. 2012. Attention, learning and the value of information. Neuron 76: 281-295. doi:10.1016/j.neuron.2012.09.034

Hearst E, Jenkins HM. 1974. Sign-tracking: the stimulus-reinforcer relation and directed action. Psychonomic Society, Austin, TX.

Hogarth L, Chase HW. 2011. Parallel goal-directed and habitual control of human drug-seeking: implications for dependence vulnerability. J Exp Psychol Anim Behav Process 37: 261-276. doi:10.1037/a0022913

Jiang YV, Sisk CA. 2019. Habit-like attention. Curr Opin Psychol 29: 65-70. doi:10.1016/j.copsyc.2018.11.014

Keefer SE, Bacharach SZ, Kochli DE, Chabot JM, Calu DJ. 2020. Effects of limited and extended pavlovian training on devaluation sensitivity of sign- and goal-tracking rats. Front Behav Neurosci 14: 3. doi:10.3389/ fnbeh.2020.00003

Kleiner M, Brainard D, Pelli D, Ingling A, Murray R, Broussard C. 2007. What's new in psychtoolbox-3. Perception 36: 1-16. doi:10.1068/ v070821

Krajbich I, Rangel A. 2011. Multialternative drift-diffusion model predicts the relationship between visual fixations and choice in value-based decisions. Proc Natl Acad Sci 108: 13852-13857. doi:10.1073/pnas 1101328108

Lelos MJ, Harrison DJ, Dunnett SB. 2011. Impaired sensitivity to Pavlovian stimulus-outcome learning after excitotoxic lesion of the ventrolateral neostriatum. Behav Brain Res 225: 522-528. doi:10.1016/j.bbr.2011.08 .017

Le Pelley ME, Pearson D, Griffiths O, Beesley T. 2015. When goals conflict with values: counterproductive attentional and oculomotor capture by reward-related stimuli. J Exp Psychol Gen 144: 158-171. doi:10.1037/ xge0000037

Le Pelley ME, Mitchell CJ, Beesley T, George DN, Wills AJ. 2016. Attention and associative learning in humans: an integrative review. Psychol Bull 142: 1111-1140. doi:10.1037/bul0000064

Le Pelley ME, Seabrooke T, Kennedy BL, Pearson D, Most SB. 2017. Miss it and miss out: counterproductive nonspatial attentional capture by task-irrelevant, value-related stimuli. Atten Percept Psychophys 79: 16281642. doi:10.3758/s13414-017-1346-1

Le Pelley ME, Pearson D, Porter A, Yee H, Luque D. 2019. Oculomotor capture is influenced by expected reward value but (maybe) not predictiveness. Q J Exp Psychol 72: 168-181. doi:10.1080/17470218 .2017.1313874

Maria-Rios C, Fitzpatrick C, Morrow JD. 2021. Manipulation of predictive and incentive value have divergent effects on sign- and goal-tracking behavior. Biol Psychiatry 89: S292-S293. doi:10.1016/j.biopsych.2021 .02 .728

Mikhael S, Watson P, Anderson BA, Le Pelley ME. 2021. You do it to yourself: attentional capture by threat-signaling stimuli persists even when entirely counterproductive. Emotion 21: 1691-1698. doi:10.1037/ emo0001003

Mine C, Saiki J. 2015. Task-irrelevant stimulus-reward association induces value-driven attentional capture. Atten Percept Psychophys 77: 18961907. doi:10.3758/s13414-015-0894-5

Morrison SE, Bamkole MA, Nicola SM. 2015. Sign tracking, but not goal tracking, is resistant to outcome devaluation. Front Neurosci 9: 468 doi:10.3389/fnins.2015.00468

Patitucci E, Nelson AJD, Dwyer DM, Honey RC. 2016. The origins of individual differences in how learning is expressed in rats: a 
general-process perspective. J Exp Psychol Anim Learn Cogn 42: 313-324. doi:10.1037/xan0000116

Pearson D, Donkin C, Tran SC, Most SB, Le Pelley ME. 2015. Cognitive control and counterproductive oculomotor capture by reward-related stimuli. Vis Cogn 23: 41-66. doi:10.1080/13506285.2014.994252

Pearson D, Osborn R, Whitford TJ, Failing M, Theeuwes J, Le Pelley ME. 2016. Value-modulated oculomotor capture by task-irrelevant stimuli is a consequence of early competition on the saccade map. Atten Percept Psychophys 78: 2226-2240. doi:10.3758/s13414-016-1135-2

Pearson D, Watson P, Albertella L, Le Pelley ME. 2022. Attentional economics links value-modulated attentional capture and decision-making. Nat Rev Psychol 1: 320-333. doi:10.1038/ s44159-022-00053-z

Pelli DG. 1997. The VideoToolbox software for visual psychophysics: transforming numbers into movies. Spat Vis 10: 437-442. doi:10.1163/ 156856897X00366

Peschel AO, Orquin JL, Mueller Loose S. 2019. Increasing consumers' attention capture and food choice through bottom-up effects. Appetite 132: 1-7. doi:10.1016/j.appet.2018.09.015

Pool ER, Brosch T, Delplanque S, Sander D. 2014. Where is the chocolate? Rapid spatial orienting toward stimuli associated with primary rewards Cognition 130: 348-359. doi:10.1016/j.cognition.2013.12.002

Pool ER, Pauli WM, Kress CS, O’Doherty JP. 2019. Behavioural evidence for parallel outcome-sensitive and outcome-insensitive Pavlovian learning systems in humans. Nat Hum Behav 3: 284. doi:10.1038/ s41562-018-0527-9

Ratcliff R. 1979. Group reaction time distributions and an analysis of distribution statistics. Psychol Bull 86: 446-461. doi:10.1037/0033-2909 86.3.446

Robbins TW, Costa RM. 2017. Habits. Curr Biol 27: R1200-R1206. doi:10 $.1016 /$ j.cub.2017.09.060

Robinson TE, Berridge KC. 1993. The neural basis of drug craving: an incentive-sensitization theory of addiction. Brain Res Brain Res Rev 18: 247-291. doi:10.1016/0165-0173(93)90013-p

Rosas JM, Todd TP, Bouton ME. 2013. Context change and associative learning. WIREs Cogn Sci 4: 237-244. doi:10.1002/wcs.1225

Rose AK, Brown K, Field M, Hogarth L. 2013. The contributions of value-based decision-making and attentional bias to alcohol-seeking following devaluation. Addiction 108: 1241-1249. doi:10.1111/add .12152

Rose AK, Brown K, MacKillop J, Field M, Hogarth L. 2018. Alcohol devaluation has dissociable effects on distinct components of alcohol behaviour. Psychopharmacology 235: 1233-1244. doi:10.1007/ s00213-018-4839-2

Salvucci D, Goldberg J. 2000. Identifying fixations and saccades in eye-tracking protocols. Proc AMIA Annu Fall Symp 71-78. doi:10.1145/ 355017.355028

Schmidt LJ, Belopolsky AV, Theeuwes J. 2015. Attentional capture by signals of threat. Cogn Emot 29: 687-694. doi:10.1080/02699931.2014.924484

Smith SM, Krajbich I. 2018. Attention and choice across domains. J Exp Psychol Gen 147: 1810-1826. doi:10.1037/xge0000482
Theeuwes J. 1992. Perceptual selectivity for color and form. Percept Psychophys 51: 599-606.

Theeuwes J, de Vries G-J, Godijn R. 2003. Attentional and oculomotor capture with static singletons. Percept Psychophys 65: 735-746. doi:10 .3758/BF03194810

Tomie A. 2018. Introduction: the role of sign-tracking in drug addiction. In Sign-tracking and drug addiction (ed. Tomie A, Morrow JD), pp. 1-7. Michigan Publishing, University of Michigan Library, Ann Arbor, MI.

van den Bergh D, van Doorn J, Marsman M, Draws T, van Kesteren E-J, Derks K, Dablander F, Gronau QF, Kucharský Š, Raj A, et al. 2020. A tutorial on conducting and interpreting a Bayesian ANOVA in JASP. Annee Psychol 120: 73-96. doi:10.3917/anpsy1.201.0073

van Strien T, Frijters JER, Bergers GPA, Defares PB. 1986. The Dutch eating behavior questionnaire (DEBQ) for assessment of restrained emotional, and external eating behavior. Int J Eat Disord 5: 295-315.

Verhoeven AAC, de Wit S. 2018. The role of habits in maladaptive behaviour and therapeutic interventions. In The psychology of habit: theory, mechanisms, change, and contexts (ed. Verplanken B), pp. 285-303. Springer, Cham, Switzerland.

Watson P, de Wit S. 2018. Current limits of experimental research into habits and future directions. Curr Opin Behav Sci 20: 33-39. doi:10.1016/ j.cobeha.2017.09.012

Watson P, Wiers RW, Hommel B, de Wit S. 2014. Working for food you don't desire. Cues interfere with goal-directed food-seeking. Appetite 79: 139_ 148. doi:10.1016/j.appet.2014.04.005

Watson P, Pearson D, Most SB, Theeuwes J, Wiers RW, le Pelley ME. 2019a. Attentional capture by Pavlovian reward-signalling distractors in visual search persists when rewards are removed. PLoS One 14: e0226284. doi:10.1371/journal.pone.0226284

Watson P, Pearson D, Wiers RW, Le Pelley ME. 2019b. Prioritizing pleasure and pain: attentional capture by reward-related and punishment-related stimuli. Curr Opin Behav Sci 26: 107-113. doi:10.1016/j.cobeha.2018.12 .002

Watson P, Pearson D, Le Pelley ME. 2020. Reduced attentional capture by reward following an acute dose of alcohol. Psychopharmacology 237: 3625-3639. doi:10.1007/s00213-020-05641-6

Watson P, Vasudevan A, Pearson D, Le Pelley ME. 2021. Eating restraint is associated with reduced attentional capture by signals of valuable food reward. Appetite 159: 105050. doi:10.1016/j.appet.2020.105050

Yantis S. 2000. Goal-directed and stimulus-driven determinants of attentional control. In Attention and performance XVIII (ed. Monsell S, Driver J), pp. 73-103. MIT Press, Cambridge, MA.

Zoltak MJ, Veling H, Chen Z, Holland RW. 2018. Attention! Can choices for low value food over high value food be trained? Appetite 124: 124-132. doi:10.1016/j.appet.2017.06.010

Received January 26, 2022; accepted in revised form June 20, 2022. 